\title{
MATHEMATICAL MODELLING IN NANOTECHNOLOGY USING CALCULUS OF VARIATIONS
}

\author{
NAWA AWAD ALSHAMMARI
}

(Received 1 November 2016; first published online 6 February 2017)

\begin{abstract}
2010 Mathematics subject classification: primary 49K10; secondary 49K15, 49K20, 49K21. Keywords and phrases: calculus of variations, curvature, nanotechnology, nanoantennas.
\end{abstract}

The purpose of this thesis is to use the classical technique known as calculus of variations to investigate three problems in nanotechnology. Curvature-dependent energies are used in modelling biological molecules including the shape of red blood cells and protein backbone structures. In the thesis, we investigate another application of such energies to model nanoantennas. Targeting mid-infrared rays, nanoantennas (which are defined as tiny gold squares or spirals set in specially treated forms of polyethylene) can convert energy into electricity giving rise to theoretical efficiency which is much higher than current solar cell technology. Since shape and size are critical to the performance of nanoantennas, here we investigate a certain type of nanoantenna which is a spherical helix or another similar curve that winds around a sphere. Using the Euler-Lagrange equations for extremal curves, we find classes of energies which permit curves on the sphere as extremisers.

The second problem is the joining between carbon nanostructures by using calculus of variations. Carbon nanostructures have received a lot of attention for their use in nanoscale devices. Joining carbon nanostructures has been shown to enhance the property of the combined structures. In the thesis we model the join of carbon nanocones with fullerenes, two nanocones, a plane sheet of graphene with nanocones, a fullerene with a plane sheet of graphene and a nanocone with two parallel sheets of graphene. We illustrate that a perfect join configuration of absolute minimum energy is possible in some circumstances. In general, we study two models, which are based on the curvature of the join profile. Model I refers to the positive curvature only and Model II refers to the positive and negative curvatures. In addition, we consider three cases of joining nanocones with exactly half a sphere, more than half a sphere and less than half a sphere. In joining two nanocones, we investigate the joining between two

Thesis submitted to the University of Wollongong in October 2015; degree approved on 3 February 2016; supervisors James McCoy and Natalie Thamwattana.

(c) 2017 Australian Mathematical Publishing Association Inc. 0004-9727/2017 \$16.00 
symmetric carbon nanocones, two different-angle nanocones and two different-angle nanocones for five possible angles of the first nanocone. These combined structures may be useful for the design of scanning tunnelling microscopy and other nanoscale devices.

Finally, the thesis compares two different energies used in modelling the joining between different carbon nanostructures. The first energy depends on the axial curvature only. As carbon nanotubes deform according to perfect elasticity, the elastic energy is used to determine the join region which contains a finite number of discrete bonds. Secondly, we consider the Willmore energy that depends on the axial and rotational curvatures. As a result, we use a piece of catenoid to join carbon nanostructures, as it is an absolute minimiser of the Willmore energy. In particular, we investigate using the two energies for joining carbon nanocones with carbon nanotubes, two carbon nanocones and two fullerenes. We conclude that both of these energies can be used for these two cases of joining, as they are similar in the joining profile.

The major contribution of the thesis is the use of applied mathematical modelling to determine optimal configurations of nanostructures. Three problems are investigated, based on geometry of space curves on a sphere, geometry of joining to combine different nanostructures and comparison of different energies for the same joining scenario.

NAWA AWAD ALSHAMMARI,

School of Mathematics and Applied Statistics, University of Wollongong, NSW 2522, Australia

e-mail: na794@uowmail.edu.au 